\title{
Stanford Seven-Day Physical Activity Recall questionnaire in COPD
}

\author{
Benjamin E. Garfield*, Jane L. Canavan*, , Cayley J. Smith*, Karen A. Ingram\#, \\ Ria P. Fowler*, Amy L. Clark ${ }^{\#}$, Michael I. Polkey* and William D-C. Man*,\#
}

ABSTRACT: Quantification of daily physical activity is of clinical interest in chronic obstructive pulmonary disease (COPD). Objective measures using activity monitors may take several days to obtain reliable results. The aim of our study was to evaluate the Stanford Seven-Day Physical Activity Recall questionnaire (PAR) against the SenseWear armband (SWA) and compare its validity with three other physical activity questionnaires.

43 COPD patients wore the SWA for 7 days. Patients completed the PAR, Baecke, Physical Activity Scale for the Elderly (PASE) and Zutphen questionnaires. Spearman rank correlation, intraclass correlation coefficients (ICC) and receiver-operating characteristics (ROC) curves were used to assess the relationship between the questionnaires and SWA.

Assessed by PAR, time spent at $\geqslant 3.0$ metabolic equivalents (METs) correlated significantly $(r=0.54, p<0.001)$ with equivalent measures from SWA, with an ICC of 0.40 . No relationship was seen between the other questionnaires and the SWA. The PAR predicted active patients ( $\geqslant 30 \mathrm{~min}$ at $\geqslant 3.0$ METs or a physical activity level $(P A L) \geqslant 1.55)$ and very inactive patients $(P A L<1.40)$ with an area under ROC curve of $0.83,0.77$ and 0.70 , respectively.

While the PAR did not measure physical activity sufficiently accurately to make individual recommendations, it was able to identify COPD patients at extremes of the physical activity spectrum, potentially reducing the number of patients requiring direct measurement.

KEYWORDS: Activity monitors, chronic obstructive pulmonary disease, physical activity, questionnaires

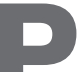
hysical inactivity is a leading cause of mortality in the western world [1]. Chronic obstructive pulmonary disease (COPD) is characterised by breathlessness and limited functional capacity. Patients often have significantly reduced daily physical activity levels compared with age-matched controls [2], even at early stages of the disease [3]. Physical activity is of prognostic significance in COPD [4]. Patients who perform some level of physical activity have lower risk of hospital admission and mortality [5], whilst those who remain inactive after a severe exacerbation have a greater risk of hospital readmission [6]. In a prospective study, moderateto-high levels of regular physical activity were associated with reduced lung function decline and COPD risk among smokers [7]. Hence, increasing daily physical activity in COPD patients and smokers is a worthwhile therapeutic aim.

Although direct observation, double-labelled water and calorimetry are considered the gold standard for assessing physical activity, they are too time consuming and expensive to be used in large population studies [8]. Activity monitors, such as accelerometers, can measure intensity and quantity of physical activity in an objective way, and have been shown to detect physical activity more accurately in sedentary populations than pedometers or subjective measures. Unfortunately, they are relatively expensive and need to be worn for several days to obtain meaningful data [9]. Furthermore, compliance with accelerometers in some studies has been poor [10].

Activity questionnaires are commonly used for physical activity assessment. The methodology is cheap allowing application to large populations. However, disadvantages include the potential for overestimating or underestimating the amount of physical activity performed [8], meaning caution should be exercised when questionnaires are used to estimate physical activity on an individual basis [11]. Despite their widespread use, no
AFFILIATIONS

${ }^{*}$ NIHR Respiratory Biomedical

Research Unit, and

\#Harefield Pulmonary Rehabilitation team, Royal Brompton and Harefield NHS Foundation Trust, London, UK.

CORRESPONDENCE

B.E. Garfield

c/o W.D-C. Man

Dept of Respiratory Medicine

Harefield Hospital

Hill End Road

Harefield

UB9 6JH

UK

E-mail: bgarfield@doctors.org.uk

Received:

July 032011

Accepted after revision:

Nov 262011

First published online:

Dec 192011 
physical activity questionnaire has been validated in patients with COPD [9].

Few studies have used objective and subjective measures of physical activity concurrently in patients with COPD. STEELE et al. [12] demonstrated no relationship between output from the Tritrac triaxial accelerometer and the Modified Activity Recall Questionnaire. Moy et al. [13] showed that an activity checklist correlated with measured steps per day, but had accurate data from only 10 of the 17 patients studied, questioning the validity of these results. Finally, one study demonstrated that time spent in different activities measured by a diary correlated significantly with step count from the Yamax digiwalker pedometer [14]. However, the diary took the same time to collect data as the inexpensive pedometer limiting its usefulness.

The Stanford Seven-Day Physical Activity Recall questionnaire (PAR) is interviewer led and is widely used in epidemiological studies [15]. This questionnaire has not previously been validated in COPD patients, although a comprehensive review did identify its potential [9]. The aim of our study was to compare four physical activity questionnaires (the PAR; BAECKE [16]; Physical Activity Scale for the Elderly (PASE) [17]; and Zutphen Physical Activity questionnaire (ZPAC) [18]), against a validated activity monitor, the SenseWear Armband (SWA), in a cohort of well-characterised patients with COPD. The SWA is a biaxial accelerometer and motion sensor that has previously been used in large cohorts of COPD patients [3, 19] and validated against indirect calorimetry [20, 21]. We hypothesised that the PAR would correlate better with output from the SWA than the other questionnaires. We further hypothesised that the PAR could be a useful stratification tool in predicting active and very inactive COPD patients.

\section{METHODS}

\section{Subjects}

45 patients with COPD were recruited from respiratory clinics at Harefield Hospital, London, UK. All patients except two had completed an 8-week outpatient pulmonary rehabilitation programme within the last 12 months. Participants were excluded if they had a diagnosis of heart, renal or liver failure, a metabolic disorder or had experienced an exacerbation in the previous 4 weeks. The study was approved by the local research ethics committee. All patients gave informed written consent.

\section{Measurements}

The study consisted of two visits, 1 week apart. At the first visit, participants underwent the following measurements: spirometry; height, weight, body mass index (BMI); 6-min walk test (6MWT) [22]; health status using the St George's Respiratory Questionnaire (SGRQ) [23]; Medical Research Council (MRC) dyspnoea scale [24]; and calculated BODE (body mass index (B), the degree of airflow obstruction $(\mathrm{O})$ and dyspnoea (D), and exercise capacity (E)) index [25]. Following assessments, patients wore the SWA over the triceps muscle of their right arm for 7 days until the next visit, as previously described [3]. At visit 2, the participants completed the interviewer-led PAR questionnaire with updated protocol (see online supplementary material) [26], and three self-complete questionnaires (PASE [27], BAECKE [28] and ZPAC [29]). Both patients and interviewer were blinded to the output of the SWA at the time of completion and analysis of the PAR.

\section{Indices of physical activity}

Participants wore the SWA $24 \mathrm{~h}$ a day except when performing any tasks that might put the armband at risk of getting wet. When $<22.5 \mathrm{~h}$ of armband use were recorded during the day, data was excluded from analysis as described by WATZ et al. [3]. Physical activity indices recorded from the SWA wereas follows. Total energy expenditure (TEE, total number of calories used per day); physical activity level (PAL, defined as total energy expenditure per minute divided by sleep energy expenditure per minute) as previously described [19]; and time spent in at least moderate physical activity (time $\geqslant 3$ metabolic equivalents (METs), defined as number of minutes per day in activity $\geqslant 3$ METs). Although the SWA also records step counts, this outcome was not used for analysis as it underestimates true step counts at low walking speeds [20].

The PAR allowed calculation of total energy expenditure and time spent in moderate (mean METs), hard (mean 6 METs) and very hard physical activity (mean $10 \mathrm{METs}$ ). From this, time spent in at least moderate activity ( $\geqslant 3$ METs) was calculated.

The PASE and BAECKE questionnaires produce a physical activity score without specific units. The outputs from the ZPAC are energy expenditure in physical activity, and time spent in light, moderate and heavy physical activity. Unlike the SWA, moderate activity is defined by ZPAC as $\geqslant 2$ METs [26]. Therefore, when comparing data output from the SWA and ZPAC the definition of time spent in moderate activity produced by the SWA was changed using the "professional" software module to time spent $\geqslant 2$ METs [26].

\section{Data analysis}

Statistical analysis was undertaken using SPSS software version 18 (SPSS Inc., Chicago, IL, USA). For a significant Pearson correlation between questionnaire and SWA at the 0.05 level, a power of $90 \%$ and a large effect size $(r=0.5)$, a minimum of 38 patients was needed. Assuming a study dropout rate of $15 \%, 45$ patients were recruited. Spearman Rank correlation was actually performed to assess the relationship between questionnaires' output and SWA physical activity indices as data was not normally distributed. When comparing time spent in moderate physical activity between SWA and the PAR and ZPAC questionnaires, Bland-Altman plots were constructed and intraclass correlation coefficients (ICC) were calculated. ICC results were interpreted using recommendations by FLEISS [30], which define ICC at $<0.40$ as poor-fair, $0.40-0.74$ as fair-good and $\geqslant 0.75$ as excellent. Receiver operator characteristics (ROC) curves and area under curve (AUC) were used to assess the ability of the questionnaires to predict "active" and "very inactive" patients with COPD. "Active" was defined in two ways: 1 ) spending at least $30 \mathrm{~min}$ in at least moderate physical activity ( $\geqslant 3$ METs) as per current recommendations [31]; and 2) achieving a PAL $\geqslant 1.55$, the mean baseline PAL of a cohort of COPD patients alive after 4 yrs of follow-up [4]. "Very inactive" was conventionally defined as a SWA-derived PAL $<1.4$ as previously described [3, $4,32]$. A binary logistic regression model was used to assess whether the PAR time $\geqslant 3$ METs offered additional information 
over 6MWT in predicting active patients with COPD. Percentage of variance explained by the model was estimated using the Nagelkerke $R^{2}$ statistic. For the two factors, $\beta$-coefficients, Wald statistics, Wald statistic significance levels and odds ratios were obtained.

\section{RESULTS}

Two of the 45 patients recruited failed to complete the study (one developed severe headaches requiring hospital admission and the other withdrew because of discomfort from wearing the SWA). Baseline characteristics of the remaining participants are outlined in table 1.

\section{SWA monitor data}

Mean daily usage was $23 \mathrm{~h}$ and $31 \mathrm{~min}$. Median (interquartile range) TEE, PAL and time $\geqslant 3$ METs were 1,910 (17072208) $\mathrm{kcal} \cdot \mathrm{day}^{-1}, 1.41(1.33-1.64)$ and $37(25-72) \mathrm{min} \cdot \mathrm{day}^{-1}$, respectively.

\section{Physical activity questionnaires}

The data generated by the questionnaires are summarised in table 2 .

\section{Relationship between physical activity questionnaires and SWA}

TEE derived by PAR correlated significantly with TEE measured by SWA $(r=0.83, p<0.001)$. Bias was 410 with $95 \%$ limits of agreements from -262 to 1,082 . PAR-derived time $\geqslant 3$ METs correlated significantly with SWA-derived time $\geqslant 3$ METs $(\mathrm{r}=0.54, \mathrm{p}<0.001)$ and with SWA-derived PAL $(\mathrm{r}=0.46$, $\mathrm{p}=0.002$ ) (fig. $1 \mathrm{a}$ and $\mathrm{b}$ ). ICC for time $\geqslant 3$ METs between PAR and SWA was 0.40 . Bias for time $\geqslant 3$ METs was 0.89 with $95 \%$ limits of agreements were from -112.9 to 114.7 (fig. 2a).

No significant association was seen between PASE score, BAECKE score or ZPAC physical activity energy expenditure with TEE or PAL derived from SWA (fig. 1c-e). The PASE score, BAECKE score and ZPAC time at $\geqslant 2$ METs did not correlate significantly with time in moderate activity derived

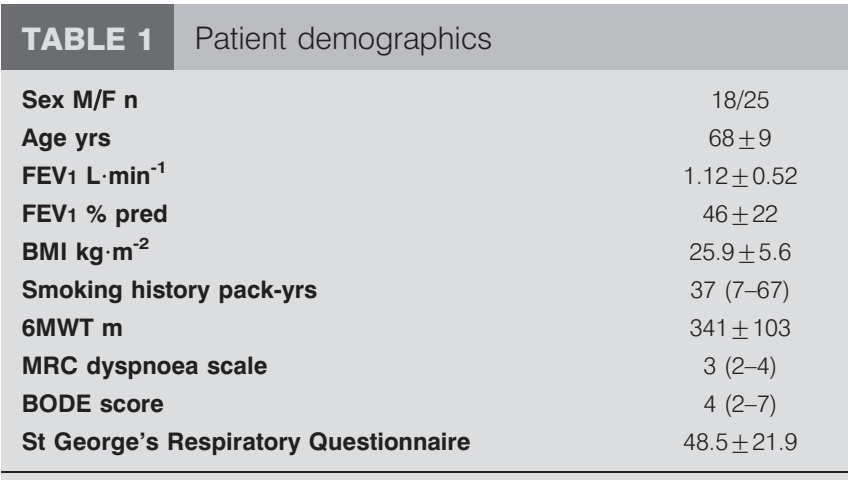

Data are presented as mean \pm SD or median (interquartile range), unless otherwise stated. M: male; F: female; FEV1: forced expiratroy volume in $1 \mathrm{~s}$; \% pred: \% predicted; BMI: body mass index; 6MWT: 6-min walk test; MRC: Medical Research Council; BODE: body mass index, degree of airflow obstruction and dyspnoea, and exercise capacity.

\begin{tabular}{|c|c|}
\hline \multicolumn{2}{|l|}{ Stanford 7-day Physical Activity Recall questionnaire } \\
\hline Total daily energy expenditure $\mathrm{kcal} \cdot \mathrm{day}^{-1}$ & $2228(1885-2897)$ \\
\hline Time in at least moderate activity min. day ${ }^{-1}$ & $36(15-69)$ \\
\hline \multicolumn{2}{|l|}{ Physical Activity Scale for the Elderly } \\
\hline Score & $122(70-156)$ \\
\hline \multicolumn{2}{|l|}{ Modified Baecke Physical Activity questionnaire } \\
\hline Score & $1.70(1.35-3.86)$ \\
\hline \multicolumn{2}{|l|}{ Zutphen Physical Activity questionnaire } \\
\hline Physical activity energy expenditure $\mathrm{kcal} \cdot \mathrm{day}^{-1}$ & $574(159-855)$ \\
\hline Time in at least moderate activity ( $\geqslant 2.0 \mathrm{METs}$ ) min. day ${ }^{-1}$ & $120(26-210)$ \\
\hline
\end{tabular}

Data are presented as median (interquartile range). METs: metabolic equivalents.

by the SWA (fig. 1f). The ICC for the ZPAC and SWA time at $\geqslant 2$ METs was 0.37 .

\section{ROC and logisitic regression}

The ROC curve for PAR predicting patients with COPD achieving at least $30 \mathrm{~min}$ of moderate activity recorded by the SWA is shown in figure 3a. A PAR time spent in physical activity ( $\geqslant 3$ METs) of $\geqslant 30 \mathrm{~min}$ had a sensitivity and specificity of 0.79 and 0.80 , respectively, and an AUC of 0.83 . With PAR time set at $\geqslant 33 \mathrm{~min}$, AUC was 0.84 with a sensitivity of 0.79 and a specificity of 0.93 . PASE, BAECKE and ZPAC had AUCs of $0.63,0.64$ and 0.57 , respectively. A $6 \mathrm{MWT}>330 \mathrm{~m}$ had an AUC of 0.72 with a sensitivity of 0.66 and a specificity of 0.64 (fig. 3a).

The ROC curve for PAR predicting a PAL $\geqslant 1.55$ is shown in figure $3 \mathrm{~b}$. A PAR time spent in $\geqslant 3$ METs of $>35 \mathrm{~min}$ had an AUC of 0.77 , a sensitivity of 0.85 and a specificity of 0.63 . PASE, BAECKE and ZPAC had AUCs of 0.64, 0.64 and 0.66, respectively. A $6 \mathrm{MWT}$ of $>330 \mathrm{~m}$ had an AUC of 0.64 , with a sensitivity of 0.69 and specificity of 0.53 (fig. 3b).

A PAR calculated time spent in $\geqslant 3$ METs of $<33 \mathrm{~min}$ had an AUC of 0.70 in predicting "very inactive" patients (PAL $<1.4$ ) with a sensitivity of 0.73 and a specificity of 0.76 (fig. 3c). PASE, BAECKE and ZPAC had AUCs of 0.60, 0.55 and 0.49, respectively. A $6 \mathrm{MWT}$ of $<330 \mathrm{~m}$ had an AUC of 0.60 in predicting very inactive patients with a sensitivity of 0.59 and a specificity of 0.61 (fig. 3c).

The PAR measured time spent in moderate physical activity (Wald Chi-squared test 5.194, p<0.05) was significant in predicting patients achieving $\geqslant 30 \mathrm{~min}$ of moderate activity measured by the SWA, with an odds ratio of 1.051 (95\% CI 1.007-1.097). This is in contrast to the 6MWT (Wald Chisquared teat 2.744, $\mathrm{p}>0.05$ ), which was not significantly able to predict active patients with an odds ratio of 1.008 (95\% CI 0.999-1.018). The Nagelkerke $\mathrm{R}^{2}$ for the model was 0.47, suggesting that $47 \%$ of the variance was accounted for. Sensitivity for the model was 0.90 and specificity was 0.71 . Percentage correct classification was $83.7 \%$.

\section{DISCUSSION}

This is the first study to simultaneously assess the validity of four physical activity questionnaires in COPD patients. Data from three of the four questionnaires bore no relationship to 

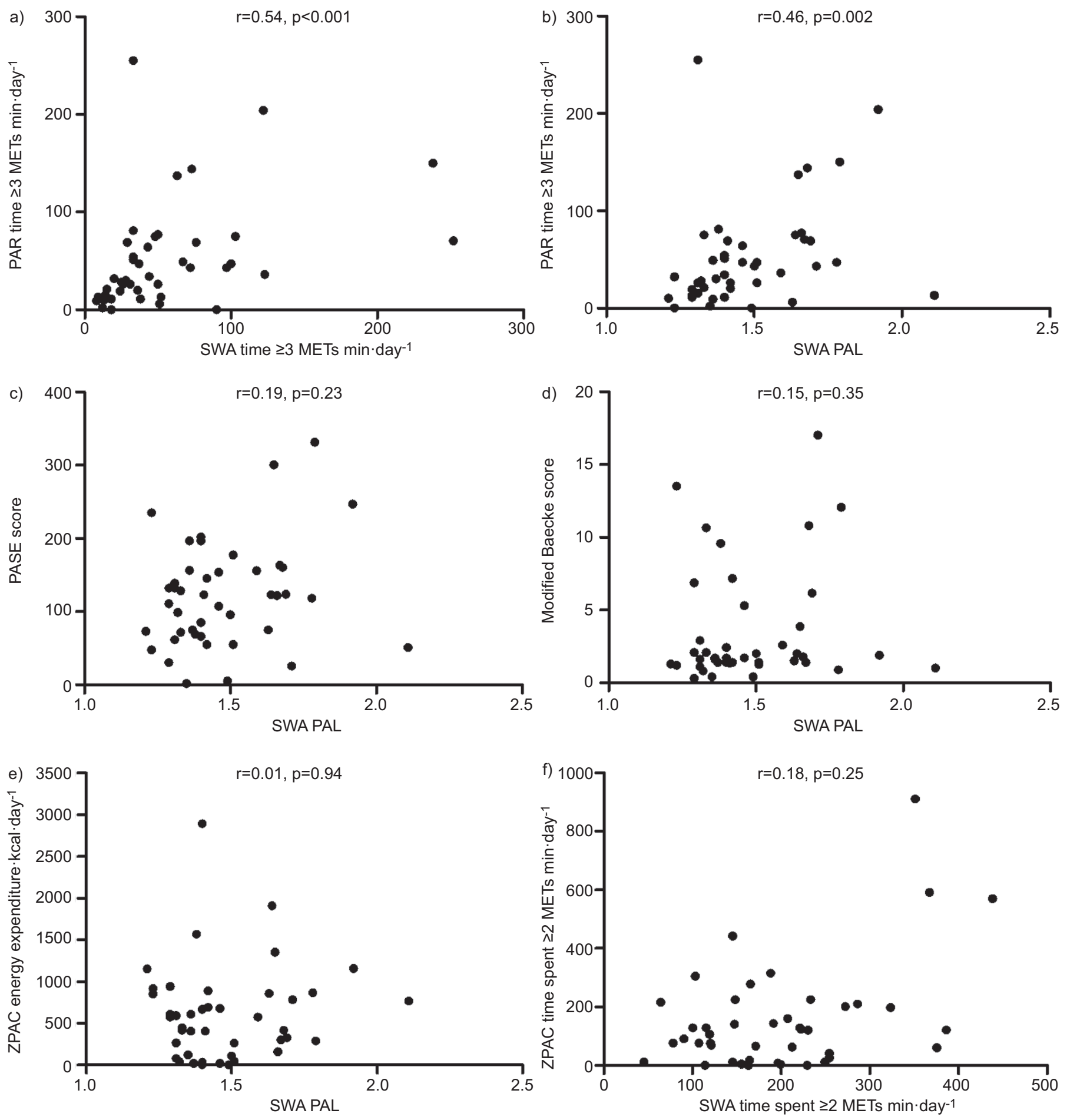

FIGURE 1. Spearman rank correlations between a) the Stanford 7-day Physical Activity Recall questionnaire (PAR) and SenseWear armband (SWA) time in $\geqslant 3$ metabolic equivalents (METs); b) PAR time $\geqslant 3$ METS and SWA physical activity level (PAL); c) Physical Activity Scale for the Elderly (PASE) score and SWA PAL; d) modified Baecke score and SWA PAL; e) Zutphen Physical Activity questionnaire (ZPAC) energy expenditure and SWA PAL; and f) ZPAC and SWA time in $\geqslant 2$ METS.

any activity index measured using a validated activity monitor. In contrast, data from the PAR questionnaire correlated significantly with equivalent indices of physical activity, although the ICC for PAR and SWA was at best fair. Furthermore, the PAR had reasonable predictive power in identifying active and very inactive COPD patients. Taken together, these results suggest that while the PAR may not be suitable for assessing physical activity on an individual level it may be a potentially useful tool for immediate stratification of COPD patients according to activity levels. 


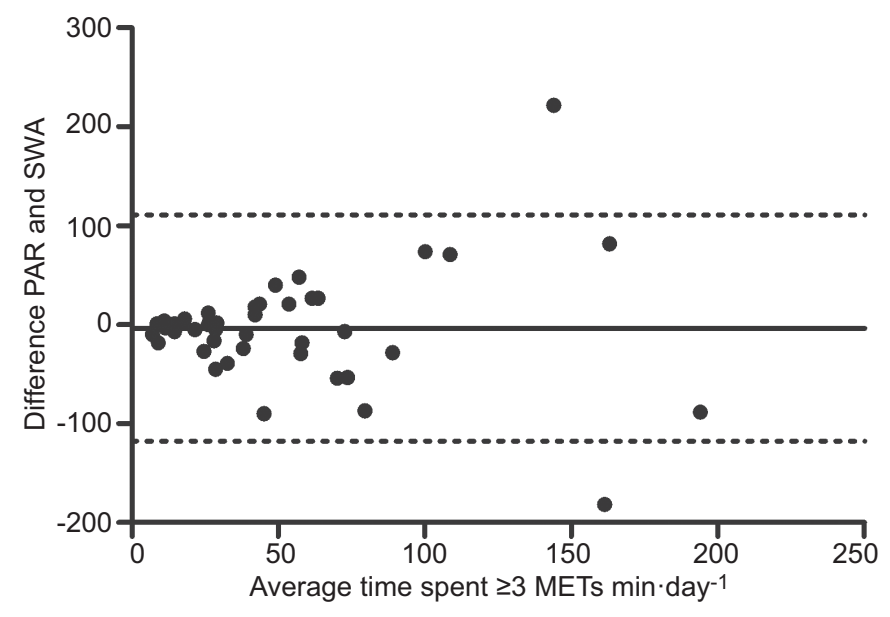

FIGURE 2. Bland-Altman plot of the average time spent at $\geqslant 3$ metabolic equivalents (METs) versus the difference in time spent in $\geqslant 3$ METs derived from the Stanford 7-day Physical Activity Recall (PAR) questionnaire and the SenseWear armband (SWA).

\section{Critique of the method}

In this study, we used data from the SWA as the criterion method to compare questionnaires. Although the traditional gold standard measurements of physical activity include direct observation, double-labelled water and calorimetry, these techniques are not possible in large numbers of patients due to expense, impracticality and patient tolerability. HiLl et al. [21] demonstrated that energy expenditure measured by SWA was sensitive to small but important changes, had good repeatability and agreed well with energy expenditure measured by indirect calorimetry. LANGER et al. [20] also demonstrated good agreement between SWA and indirect calorimetry in measuring energy expenditure and time spent in at least moderate physical activity ( $\geqslant 3 \mathrm{METs}$ ). Our cohort was sedentary with a median physical activity level of 1.41 . They spent a median of $37 \mathrm{~min}$ per day in at least moderate physical activity. Mean daily use of SWA was $>23 \mathrm{~h}$. To date, these results are comparable with the data from the largest cohort of COPD patients using the SWA [3, 19].

This study has the usual limitations of cross-sectional studies. First, our cohort had moderate-severe disease (mean forced expiratroy volume in $1 \mathrm{~s}$ was $46 \%$ predicted), so the results may not be applicable to the general COPD population; indeed limits of agreement widened as function improved. Secondly, our patients were in the stable state and, hence, our study cannot shed light on what might happen during recognised triggers of physical inactivity, such as acute exacerbations. Thirdly, as mentioned previously, our study did not assess the reliability or responsiveness to change of the questionnaires.

\section{PAR questionnaire}

The PAR is a semi-structured interview that was originally developed in the early 1980s [15] and has since been widely used in clinical studies. To our knowledge, the PAR has been used once in a small study of patients with COPD [33]. There has been no previous attempt to validate the PAR in COPD patients, but data from other populations support its validity. In a validation study of five questionnaires against a uniaxial accelerometer, the PAR had the best correlation coefficient [34]. Furthermore, in a validation study of 10 questionnaires completed by healthy elderly males, the PAR correlated significantly with total energy expenditure measured by double-labelled water and compared favourably with the other questionnaires [35].

We found a significant (although relatively modest) correlation between output from the PAR and equivalent measures from the SWA in our COPD cohort. This is in contrast to the other three questionnaires evaluated. This may reflect the interviewled, semi-structured nature of the PAR in comparison with the other questionnaires, which were self-completed and unsupervised. The PAR adheres to the cognitive model of question response. The comprehension and decision making stages are aided by the use of unambiguous questions with clearly defined time scales and activity levels. Guided memory techniques are used to minimise recall error [36].
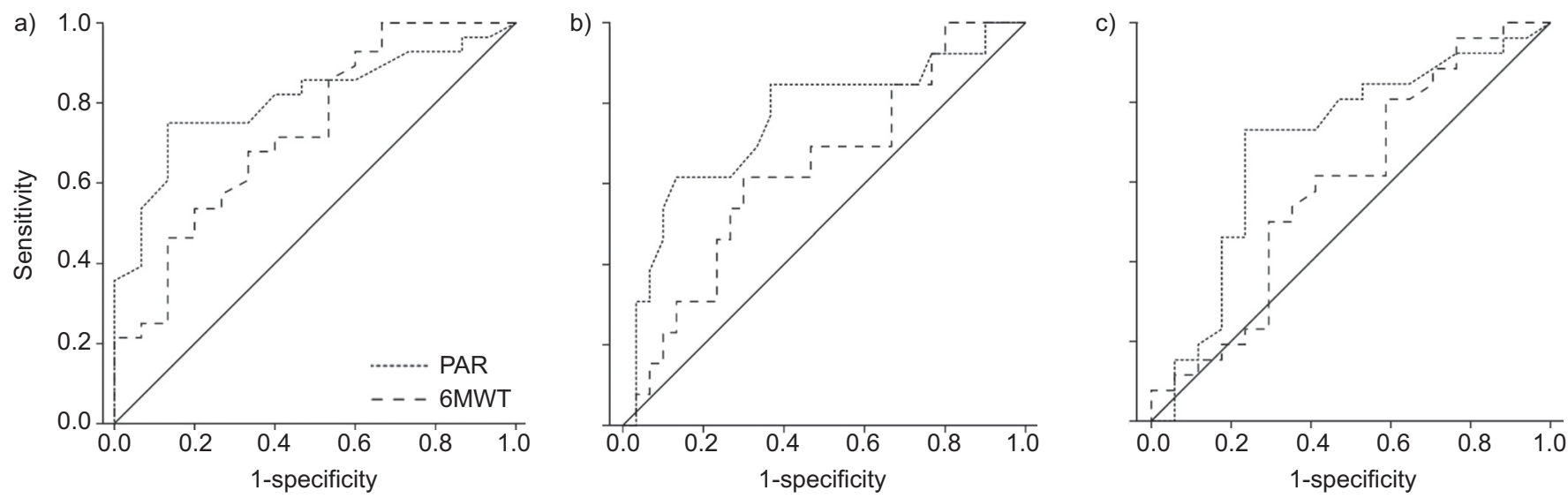

FIGURE 3. Receiver operating characteristic (ROC) curves for a) the Stanford Seven-Day Physical Activity Recall questionnaire (PAR) and 6-min walk test (6MWT) serving as a predictor of active patients with chronic obstructive pulmonary disease (COPD) (defined as $\geqslant 30$ min per day in physical activity of $\geqslant 3$ metabolic equivalents); b) ROC curves for the PAR and 6MWT serving as a predictor of active patients with COPD with increased survival rates (physical activity level (PAL) $\geqslant 1.55$ ); and c) ROC curves for the PAR and 6MWT serving as a predictor of very inactive patients with COPD (PAL <1.4). 
In clinical trial design, the sample size (and costs) can be reduced if the outcome measure occurs frequently in the study population. Since physical inactivity is associated with mortality [4], then a therapeutic trial that used mortality as an outcome could be performed more efficiently if the participants were restricted to those who were physically inactive. In the current study, the PAR had a reasonably good sensitivity and specificity in predicting both active and very inactive COPD patients. This suggests that it may have a potential role in stratifying COPD patients for clinical trials, either directly or as a screen prior to objective measurement. As a stratification tool, the PAR has some advantages over more objective measures of physical activity. First, it is cheap in comparison to most accelerometers used in COPD studies [9]. Although we did not make a formal economic evaluation, apart from the cost of the SWA itself, other associated costs include a personal computer and the necessary software, as well as investigator time required to measure basic anthropometric measurements, input patient details into the SWA and calculate the PAL (which is not automated by the current software). Secondly, it is a quick method of assessment. The PAR was generally completed within $15 \mathrm{~min}$ in our cohort. This is in comparison with a minimum of five consecutive days of measurement required for the SWA [3]. Thirdly, calculation of the data did not require any expensive software, as is the case with some activity monitors [9]. Finally, there was a $100 \%$ completion rate of the PAR. Although the SWA was very well tolerated in this and previous studies [3], patient compliance and technical issues have been noted with some activity monitors [10, 13].

Some argue that functional measures, such as 6MWT, may predict physical activity with similar accuracy to physical activity questionnaires, such as the PAR. In our study cohort, the PAR had a better combined sensitivity and specificity, and a higher AUC than 6MWT. Furthermore, the model describing a combination of 6MWT and PAR suggested that only the PAR was an independent predictor of active patients, lending support to the hypothesis that indirect measures of physical activity are epiphenomenon. They can also provide no information on the intensity or domain of physical activity the patient performed.

\section{Conclusions}

In summary, the estimation of physical activity by the PAR correlates with data from a validated activity monitor in COPD patients, and had a reasonably good sensitivity and specificity in identifying active and very inactive COPD patients. The PAR, although unlikely to be able to measure PA usefully on an individual basis, may be a useful tool to stratify COPD patients according to their level of physical activity.

\section{STATEMENT OF INTEREST}

Statements of interest for C.J. Smith and M.I. Polkey can be found at www.erj.ersjournals.com/site/misc/statements.xhtml

\section{ACKNOWLEDGEMENTS}

W.D-C. Man is funded by a National Institute for Health Research (NIHR) Clinician Scientist award and a Medical Research Council (MRC) New Investigator Research Grant. This project was undertaken at the NIHR Biomedical Research Unit at the Royal Brompton and Harefield NHS Foundation Trust and Imperial College London. J.L. Canavan's salary is fully funded and M.I. Polkey's salary is partly funded by the Biomedical Research Unit. C.J. Smith's salary during this period was supported by a grant from GlaxoSmithKline. The views expressed in this publication are those of the authors and not necessarily those of the National Health Service, the NIHR or the Department of Health.

\section{REFERENCES}

1 Mokdad AH, Marks JS, Stroup DF, et al. Actual causes of death in the United States, 2000. JAMA 2004; 291: 1238-1245.

2 Pitta F, Troosters T, Spruit MA, et al. Characteristics of physical activities in daily life in chronic obstructive pulmonary disease. Am I Respir Crit Care Med 2005; 171: 972-977.

3 Watz H, Waschki B, Meyer T, et al. Physical activity in patients with COPD. Eur Respir J 2009; 33: 262-272.

4 Waschki B, Kirsten A, Holz O, et al. Physical activity is the strongest predictor of all-cause mortality in patients with COPD: a prospective cohort study. Chest 2011; 140: 331-342.

5 Garcia-Aymerich J, Lange P, Benet M, et al. Regular physical activity reduces hospital admission and mortality in chronic obstructive pulmonary disease: a population based cohort study. Thorax 2006; 61: 772-778.

6 Garcia-Aymerich J, Farrero E, Felez MA, et al. Risk factors of readmission to hospital for a COPD exacerbation: a prospective study. Thorax 2003; 58: 100-105.

7 Garcia-Aymerich J, Lange P, Benet M, et al. Regular physical activity modifies smoking-related lung function decline and reduces risk of chronic obstructive pulmonary disease: a population-based cohort study. Am J Respir Crit Care Med 2007; 175: 458-463.

8 Westerterp KR. Assessment of physical activity: a critical appraisal. Eur J Appl Physiol 2009; 105: 823-828.

9 Pitta F, Troosters T, Probst VS, et al. Quantifying physical activity in daily life with questionnaires and motion sensors in COPD. Eur Respir J 2006; 27: 1040-1055.

10 Kochersberger G, McConnell E, Kuchibhatla MN, et al. The reliability, validity, and stability of a measure of physical activity in the elderly. Arch Phys Med Rehabil 1996; 77: 793-795.

11 Pitta F, Troosters T, Probst VS, et al. Quantifying physical activity in daily life with questionnaires and motion sensors in COPD. Eur Respir J 2006; 27: 1040-1055.

12 Steele BG, Belza B, Hunziker J, et al. Monitoring daily activity during pulmonary rehabilitation using a triaxial accelerometer. J Cardiopulm Rehabil 2003; 23: 139-142.

13 Moy ML, Matthess K, Stolzmann K, et al. Free-living physical activity in COPD: assessment with accelerometer and activity checklist. J Rehabil Res Dev 2009; 46: 277-286.

14 Moore R, Berlowitz D, Denehy L, et al. Comparison of pedometer and activity diary for measurement of physical activity in chronic obstructive pulmonary disease. J Cardiopulm Rehabil Prev 2009; 29: 57-61.

15 Blair SN, Haskell WL, Ho P, et al. Assessment of habitual physical activity by a seven-day recall in a community survey and controlled experiments. Am J Epidemiol 1985; 122: 794-804.

16 Coronell C, Orozco-Levi M, Mendez R, et al. Relevance of assessing quadriceps endurance in patients with COPD. Eur Respir J 2004; 24: 129-136.

17 Gosker HR, Lencer NH, Franssen FM, et al. Striking similarities in systemic factors contributing to decreased exercise capacity in patients with severe chronic heart failure or COPD. Chest 2003; 123: $1416-1424$.

18 Slinde F, Ellegard L, Gronberg AM, et al. Total energy expenditure in underweight patients with severe chronic obstructive pulmonary disease living at home. Clin Nutr 2003; 22: 159-165.

19 Watz H, Waschki B, Boehme C, et al. Extrapulmonary effects of chronic obstructive pulmonary disease on physical activity: a cross-sectional study. Am J Respir Crit Care Med 2008; 177: 743-751. 
20 Langer D, Gosselink R, Sena R, et al. Validation of two activity monitors in patients with COPD. Thorax 2009; 64: 641-642.

21 Hill K, Dolmage TE, Woon L, et al. Measurement properties of the SenseWear armband in adults with chronic obstructive pulmonary disease. Thorax 2010; 65: 486-491.

22 ATS statement. guidelines for the six-minute walk test. Am J Respir Crit Care Med 2002; 166: 111-117.

23 Jones PW, Quirk FH, Baveystock CM, et al. A self-complete measure of health status for chronic airflow limitation. The St. George's Respiratory Questionnaire. Am Rev Respir Dis 1992; 145: 1321-1327.

24 Fletcher CM, Elmes PC, Fairbairn AS, et al. The significance of respiratory symptoms and the diagnosis of chronic bronchitis in a working population. BMJ 1959; 2: 257-266.

25 Celli BR, Cote CG, Marin JM, et al. The body-mass index, airflow obstruction, dyspnea, and exercise capacity index in chronic obstructive pulmonary disease. N Engl J Med 2004; 350: 1005-1012.

26 Pereira MA, FitzerGerald SJ, Gregg EW, et al. A collection of physical activity questionnaires for health-related research. The seven-day physical activity recall. Med Sci Sports Exerc 1997; 29: 89-103.

27 Washburn RA, Smith KW, Jette AM, et al. The physical activity scale for the elderly (PASE): development and evaluation. J Clin Epidemiol 1993; 46: 153-162.

28 Voorrips LE, Ravelli AC, Dongelmans PC, et al. A physical activity questionnaire for the elderly. Med Sci Sports Exerc 1991; 23: 974-979.
29 Caspersen CJ, Bloemberg BP, Saris WH, et al. The prevalence of selected physical activities and their relation with coronary heart disease risk factors in elderly men: the Zutphen Study, 1985. Am J Epidemiol 1991; 133: 1078-1092.

30 Fleiss JL. The Design and Analysis of Clinical Experiments. Wiley, New York; 1986.

31 Haskell WL, Lee IM, Pate RR, et al. Physical activity and public health: updated recommendation for adults from the American College of Sports Medicine and the American Heart Association. Circulation 2007; 116: 1081-1093.

32 Manini TM, Everhart JE, Patel KV, et al. Daily activity energy expenditure and mortality among older adults. JAMA 2006; 296: 171-179.

33 Faulkner J, Walshaw E, Campbell J, et al. The feasibility of recruiting patients with early COPD to a pilot trial assessing the effects of a physical activity intervention. Prim Care Respir J 2010; 19: $124-130$

34 Miller DJ, Freedson PS, Kline GM. Comparison of activity levels using the Caltrac accelerometer and five questionnaires. Med Sci Sports Exerc 1994; 26: 376-382.

35 Bonnefoy M, Normand S, Pachiaudi C, et al. Simultaneous validation of ten physical activity questionnaires in older men: a doubly labeled water study. J Am Geriatr Soc 2001; 49: 28-35.

36 Sharrack B, Hughes RA. Reliability of distance estimation by doctors and patients: cross sectional study. BMJ 1997; 315: $1652-1654$. 